\title{
The Master SN curve approach - A hybrid multi-scale fatigue simulation of short fiber reinforced composites
}

Atul Jain ${ }^{*}, 1,2, \neq$, Jose M. Veas ${ }^{2}$, Stefan Straesserl, Wim Van Paepegem ${ }^{3}$, Ignaas Verpoest ${ }^{2}$, Stepan V. Lomov ${ }^{2}$

1. Siemens Industry Software NV, Interleuvenlaan 68, B-3001 Leuven, Belgium

2. Department of Materials Engineering, KULeuven, Belgium

3. Department of Material Science and Engineering, Ghent University, Belgium

*corresponding author: atul.jain@mtm.kuleuven.be / atul.jain@ siemens.com

* Current affiliation: University of Southern California, Los Angeles, USA, email atuljain@usc.edu

ABSTRACT: Typical short fiber reinforced composites (SFRCs) components have a different statistical distribution of orientation of fibers at different points leading to different static and fatigue behavior at different locations across the component. To link component-scale calculations with this variability of fiber orientations, each element in the FE model is modelled as a Representative Volume Element (RVE); the static and fatigue properties must be calculated for each of these elements. While there are established methods to estimate the static properties, there are none for the fatigue properties. A hybrid (combination of micromechanics and tests) and multi-scale (damage in micro-scale linked to macroscale fatigue properties) method of predicting the SN curve for every point in a short fiber composite has been developed. This proposed method is based not only on tests but on a combination of manufacturing simulation, tests and multi-scale mechanics. An extensive test program was undertaken to study the fatigue behavior of short fiber composites and validate the concept of the Master SN curve (MSNC) approach. The MSNC approach is compared with two 
prevalent approaches - strength based scaling and test based interpolation. The MSNC approach was found to be in a good agreement with the experimental results and was confirmed to be more accurate than the prevalent methods.

\section{Introduction and motivation}

Increasingly short fiber reinforced composites (SFRC) are being looked at as possible replacements for metals in semi-structural applications in automobiles. This is due to the rising awareness and strict regulations towards reducing $\mathrm{CO}_{2}$ emissions. Additionally, these materials are cost effective since they have good specific properties and have low cost of manufacturing. Lack of proper methods for fatigue simulation has often been seen as a bottleneck for large scale deployment of SFRCs in industrial use. SFRCs are usually manufactured by the injection molding process and have a different local statistical distribution of fiber orientation at every point. Fatigue properties of SFRCs are known to depend on the fiber orientation distribution (FOD) and fiber length distribution (FLD) of the fibers [1-4], but the exact relationship is unknown. This is because unlike the static properties which are well understood; the understanding of the fatigue behavior of SFRCs is still rather limited.

For a component-level simulation, each point in the FE model has a known statistical distribution of length and orientation of fibers which are often predicted by using manufacturing simulation tools. Each point in the FE mesh can therefore be imagined as a different material whose static and fatigue properties need to be estimated [5]. A schematic representation of the component level simulation of SFRCs is shown in Figure 1.

Starting from the mold geometry, a manufacturing simulation of injection molding is usually performed to calculate the $2^{\text {nd }}$ order orientation tensor at every point. This data 
is then transferred to homogenization software which calculates the effective stiffness at that point. The effective properties at every point must then be transferred to the FE and fatigue solver. A mapping tool ensures that the mesh of the manufacturing simulation software is mapped appropriately to the FE and fatigue solver. Apart from the stiffness of the RVE, the SN curve(s) must also be calculated; this aspect of the component level simulation is studied in this paper.

For an RVE, one can calculate the effective static properties of the composite by a number of different methods, for example the Mori-Tanaka (MT) formulation [6]. However, unlike quasi-static behavior there are no well understood and universally accepted formulations to describe composite fatigue, thus there is an absence of an analytical method to predict the SN curve of a composite material. This paper proposes a method to estimate the local SN curves of a SFRCs component based on only one SN curve as input.

The standard practice in fatigue simulation of SFRCs is to make simplifying assumptions about the orientation and the length distribution of the fibers and use test based interpolation methods. Such methods depend on a number of tests on coupons with extreme FOD [7]. Furthermore, since it is almost impossible to create test coupons with perfect extreme alignment some extrapolation might be needed as well. Other approaches include use of empirical reduction coefficients for different factors like FOD, length, notch, mean stress etc. Such an approach is often used for polymers [8]. A similar approach which requires extremely large number of tests and lacks physical basis was tried by Guster et al. [9] and Mosenbacher et al. [10] for the simulation of SFRCs.

Another practical approach is to normalize the fatigue strength based on the ultimate tensile strength (UTS). This approach is based on experiments that suggest the 
proportionality of both properties in some cases[1-3,11,12]. Such an approach has two major limitations, first there is no physical reasoning as to why the fatigue strengths must vary in the same manner as the tensile strength, there are experimental evidences which confirm that the failure mechanisms for damage propagation and final failure are different during tensile and fatigue loading $[11,13,14]$. The phenomena near final failure (catastrophic loss of load-carrying ability) during tensile failure are different from the phenomena during "slow" loss of load-carrying ability during cyclic loading. Also the damage mechanisms of some glass fiber reinforced semi-crystalline polymers could depend on the strain rate $[15,16]$. And secondly, to be able to use this method one has to either model tensile strengths of SFRCs or experimentally derive the UTS for every orientation. To the best of our knowledge, there are no known reliable methods to predict the tensile strength of SFRCs accurately.

There are some studies which are based on a fracture mechanics approach have been proposed and tried for prediction of fatigue behavior with varying degrees of success $[3,17]$. Apart from a SN curve, the input for such an approach is a fatigue crack growth parameter. Experimental tests to derive the fatigue crack growth parameter are quite complicated. Damage leads to energy dissipation and energy dissipation leads to increase in heat generation. Based on this principle, Meneghetti et al. [18] and Jegou et al. [19] proposed a method for SN curve prediction of SFRCs based on the specific heat dissipation, such an approach requires rather extensive and difficult tests.

Thus one can conclude that there are a number of methods to estimate the local SN curve of a SFRCs component. However, all of them depend on a number of input parameters and also sometimes lack physical basis.

This paper aims to relate the damage at the microscopic level to the macroscopic fatigue properties and propose a hybrid multi-scale approach - the Master SN curve (MSNC) 
approach. The key innovative idea in this process is that it combines test results and simulation results (hybrid approach) on different scales (microscopic simulation, macroscopic fatigue behavior), overcoming problems of depending on too many expensive tests (pure test-based macroscopic approach) and lack of efficiency (pure microscopic simulation approach).

Problems in fatigue like many other engineering problems offer two different routes that could be considered. The first is that of a scientist who is interested in understanding reality with the highest possible accuracy and developing models to describe his understanding. The second is that of an engineer who needs tools to safely design parts which are subject to complex load histories. These two routes are usually contrasting in nature [20]. In this paper the two contrasting approaches are balanced. While the overall goal is to develop a model and a tool for fatigue simulation of SFRCs; the understanding of fatigue and damage mechanisms was also given due importance.

Section 2 of this paper describes the formulation of the Master SN curve approach (MSNC). Section 3 is devoted to the description of the experiments and the numerical implementation. The results are shown in section 4 and the conclusions are derived in section 5 .

\section{Theory and formulation of the Master SN curve approach}

As described in the previous section, the common method of scaling the SN curve on the basis of the UTS has some limitations. Another possible approach could be to scale the SN curve based on the endurance strength of SFRCs. However estimating the endurance strength of polymer composites is not an easy task. This is because unlike metals, a single crack does not propagate to cause failure during cyclic loading. The stress values at the onset of damage are typically lower than the expected endurance 
strength [21]. It is known that the first mode of damage in SFRCs is fiber matrix debonding. There are multiple methods to calculate the onset of debonding, for example the Modified Coulomb criteria [22]. It can be shown that the stress at which onset of debonding occurs is much lower than the expected stress to failure for a SFRCs after a million cycles of loading. This is consistent with reported findings for long glass and carbon fiber composites [21]. Like all composite materials, fatigue failure of SFRCs generally occurs when cracks propagate sufficiently enough to cause final failure. Under uniform cyclic loading, single fiber matrix debonds cannot propagate sufficiently to cause failure of SFRCs. The extent of damage in SFRCs must be sufficiently big to propagate and cause final failure.

In the proposed MSNC approach the extent of damage is quantified by a damage parameter. This damage parameter is defined as the loss of stiffness in the applied loading direction. Mathematically, the damage parameter is defined by the following relation:

$E_{1}=E_{0}(1-d)$

where, $E_{1}$ is the secant modulus of the RVE in the axial direction after the first half cycle of load, while $E_{0}$ is the initial Young's modulus and $d$ is the value of the damage parameter.

It is then assumed that for SFRCs with different FOD (and FLD) but same constituents (fiber and matrix), the extent of damage (which is quantified by a damage parameter) needed to propagate and cause failure after same number of cycles should be similar. The expected damage events happening at the micro-level are expected to be similar, provided the FOD of the material is sufficiently random. 
In other words, it is assumed that the damage propagation (and subsequent loss of stiffness) during cyclic loading in SFRCs is similar for the same number of cycles to failure. It is known that RVE's with different FOD and different FLD have a different stress to failure for a certain number of cycles. But the stiffness degradation curve is similar. This is the key assumption of the MSNC approach. There are indications that this assumption is reasonable and was confirmed by De Monte et al. [2]. De Monte et al. showed that the stiffness degradation was similar for coupons with similar FOD and different with different FLD.

Typical loss of stiffness curves for SFRC coupons made out of 35\% volume glass fiber reinforced polybutylene terephthalate with different FOD are presented in Figure 2. The assumption made during the MSNC approach is that the difference in the loss of stiffness curves for different FOD for similar number of cycles to failure is not significant and that for analysis purpose they can be treated as being same.

A statistical treatment of the loss of stiffness curves will involve the linearizing of the loss of stiffness curves and then comparing the slopes and the intercept of the loss of stiffness curves by some statistical method for example analysis of variance (ANOVA). This analysis will be presented in another paper [23].Based on the reference $\mathrm{SN}$ curve, the values of the damage parameter are calculated for the strength to failure for the number of cycles corresponding to different values of number of cycles to failure. It is then assumed that for a given number of cycles to failure, different RVE's may have different strength to failure for the number of cycles, but the damage parameter values corresponding to the number of cycles to failure are the same. Thus for different RVE's, for a given number of cycles to failure, the strength to failure for the number of cycles is estimated by calculating the stress levels required to attain the same value of the damage 
parameter as was calculated from the input $\mathrm{SN}$ curve. Using this principle, the points in the SN curves of different RVE can be estimated.

Another possible interpretation of the MSNC is that when there are no exact methods to estimate the failure of a composite, a practical approach to model the tensile failure is to assume failure when a certain predefined percentage of stiffness is lost. The proposed MSNC equates the loss of stiffness to generate the SN curve for the target RVE. Therefore the MSNC can also be interpreted along similar lines as scaling the SN curve based on the UTS. However unlike scaling on the basis of UTS, the MSNC approach is based on equating lower values of loss of stiffness (corresponding to the strength in the chosen point in the input SN curve). One can expect that the error in the MSNC predictions will be less as compared to the error while scaling on the basis of UTS. This is because the uncertainty in the modelling of the final tensile failure has been removed. This claim is later proved in section 4.4.

A step by step algorithm for the MSNC approach is given below:

Step 1: Number of cycles to failure and corresponding peak stress, S1 value, is extracted from the reference SN curve

Step 2: The initial Young's modulus of the test coupon, $E_{0}$ is calculated by the MT formulation

Step 3: Uniaxial loading is simulated till the peak stress $S 1$ by the MT formulation; non-linear response is simulated by treating fiber matrix debonding and matrix nonlinearity

A brief description of the treatment of fiber-matrix debonding and matrix non-linearity is provided later in this section. 
Step 4: The Young's modulus of the reference test coupon after applied load, $E_{1}$ is calculated.

Step 5: The damage parameter for the first cycle, $d$ is calculated based on Eq. 1 .

Step 6: The initial Young's modulus of the target RVE (RVE whose SN curve is to be determined) is calculated.

Step 7: Small load increments are applied to the target RVE. The stiffness and the damage parameter are calculated at the end of every time step.

Step 8: Step 7 is repeated till the value of damage parameter is equal to $d$. The stress at which the same damage parameter is reached, S2, is the stress to failure for the chosen $\mathrm{N}$ number of cycles for the target RVE.

Step 9: Step 1-8 is repeated for as many points as is needed.

A schematic flowchart of the MSNC approach for one data point is given in Figure 3. Apart from the elastic properties of the fiber and the matrix and the stress strain curve of the pure matrix, only one $\mathrm{SN}$ curve is needed for this analysis.

In the proposed MSNC approach, the first cycle of loading is modelled by using the full MT formulation, which is chosen based on its predictive abilities for stresses in individual inclusions [24] and the degree of asymmetry in the predicted stiffness tensor is also rather limited when the MT formulation is applied to SFRCs [25]. Non-linear stress strain behavior of SFRCs during the first cycle of loading is modelled by accounting for fiber-matrix debonding and matrix non-linearity, which is described below.

Onset of fiber-matrix debonding is calculated by the Modified Coulomb criteria. Fibers with debonded interface cannot be treated directly by the MT formulation. This is 
because the MT formulation is based on the Eshelby tensor, which assumes perfect bonding between the fiber and the matrix. Fibers with debonded interface are replaced by a fictitious "Equivalent Bonded Inclusion" $(E q B I)$ so that the Eshelby tensor and subsequently the effective stiffness of the SFRCs can be calculated by the MT formulation. The properties of the EqBI are calculated based on the knowledge that the stress distribution in the fiber changes due to the presence of debonded interface.

The diagonal terms, $C^{\prime}{ }_{i i}$ of the stiffness tensor of the $E q B I$ are determined as a product of (i) the corresponding diagonal terms $\left(C_{i i}\right)$ of the stiffness tensor of the original inclusion and (ii) the ratio of the average stress in the inclusion with debonded interface $<\sigma_{i i}^{\prime}>$ to the average stress that would be built in the inclusion, if it was perfectly bonded $<\sigma_{i i}>$ :

$C^{\prime}{ }_{i i}=\frac{\left.<\sigma_{i i}^{\prime}\right\rangle}{<\sigma_{i i}>} C_{i i}$

where $C^{\prime}{ }_{i i}, C_{i i}$ are the stiffness component of the $E q B I$ and the original inclusion which relates the average stress $\sigma_{i i}$ in a composite to the applied strain $\varepsilon_{i i}, \sigma_{i i}^{\prime}$ and $\sigma_{i i}$ are stresses in the inclusion with debonded and perfect interface respectively; $<>$ indicates volume averaging. Ideally the non-diagonal terms of the $E q B I, C_{i j}$ must be calculated based on the reduced average of the stress component $\sigma_{j j}$ when the applied strain is $\varepsilon_{i i}$. However formulations to calculate the stress in transverse direction of an inclusion (both bonded and debonded) when subjected to strain loading in the axial direction are unavailable. The non-diagonal terms of the stiffness tensor are calculated assuming that the Poisson's ratio of the inclusion does not change. By this method it can be ensured that the effective stress response of the two systems i.e. the RVE with EqBI and the original RVE with fibers having debonded interface is the same.

In this scheme, the tip debonding (Type A debonding) and debonding in other places (Type B debonding) is treated differently and expressions for the redistribution of the 
stresses due to debonded interface when subjected to different loads are derived. For a detailed description of the expressions for the stress redistribution due to debonded interface and a FE based validation of the mechanical equivalence the readers are referred to [22]. Matrix non-linearity is calculated based on the secant modulus approach. In this approach the modulus of the matrix subject to a given strain is calculated on the basis of the stress-strain curve of the pure matrix.

\section{Experiments and numerical implementation}

Static and fatigue experiments are performed to validate the MSNC approach. SN curves are generated for SFRCs coupons with three different orientations.

\subsection{Material and experimental set up}

To create coupons for the tensile and fatigue tests, pre-compounded polybutylene terephthalate (PBT) reinforced with 50\% weight fraction of glass fiber (equivalent volume fraction is 0.35 ) was used. The FLD of such materials can be affected by fiber breakage during the compounding process, but such information is rarely available, rather only the average fiber length is typically shared by the material supplier.

The material was injection molded to plates having dimensions $170 \times 170 \times 2 \mathrm{~mm}$. The thickness of the plates are chosen to be as thin as possible so as to ensure uniform FOD (with negligible core layer) through the thickness of the coupon based on the advice of Vincent et al. [26].

Coupons were machined from the plates in three directions, inclined at angles $\Phi=0,45$ and 90 with respect to the prevailing flow direction (Figure 4). Three coupons were made per plate for the 0 and 90 degree coupon while only one coupon was made per plate for the 45 degree coupons (as shown in the Figure 4b). For the rest of the paper, they are referred to as 0,45 and 90 degree coupon respectively. The Young's modulus for the glass fiber and the matrix was given to be 72 and 2.6 GPa respectively while the 
Poisson's ratio is 0.22 and 0.37 , and the yield strength of the matrix is taken to be 55 $\operatorname{MPa}[27]$

The manufacturing simulation was done to calculate the fiber orientation distribution of the coupons. The manufacturing simulation software SIGMASOFT [28] was used for the simulations.

Particular attention was paid to the region comprising the gauge length of the coupon. Simulation results showed that there was very little variation of the 2 nd order orientation tensor in the central part of the specimen, where gauge lengths of all coupons fit. The variation in the predicted value of the tensor component $\mathrm{a}_{11}$ was found to be less than 0.02 . For numerical simulation the entire coupon could be treated as a single RVE, whose orientation distribution of inclusions is described by the second order orientation tensor predicted by the manufacturing simulation. The orientation tensor of the 0-degree coupon was found to be

$$
a_{i j}=\left[\begin{array}{ccc}
0.81 & 0.018 & 0.137 \\
0.018 & 0.11 & 0.004 \\
0.137 & 0.004 & 0.079
\end{array}\right]
$$

The orientation tensor of the 45 and 90-degree coupons were calculated by rotating of the $0^{\circ}$ orientation tensor. The aspect ratio of the fibers is taken to be constant and equal to 20 (length of about 200 microns with a diameter of about 10 microns).

Before the fatigue tests, quasi-static tensile tests (with displacement rate of $0.5 \mathrm{~mm} / \mathrm{min}$ ) were performed on the three samples and the value of the ultimate tensile strength (UTS) was derived. This serves two purposes: First, the value of the UTS is used for getting an idea about the possible fatigue strengths, this will aid in performing the fatigue tests. Also, the value of the UTS will be used to implement the UTS based scaling and subsequent comparison with the proposed MSNC approach. 5 tensile tests were performed per sample to get a reliable value of the UTS. 
Fatigue tests were performed using a hydraulic horizontal Schenck fatigue testing machine for the 0,45 and 90-degree coupons. The machine was equipped with a load cell of $10 \mathrm{kN}$. Tests were load controlled and a sinusoidal load function with constant amplitude was applied. The applied loads had an R-ratio of 0.1 (R-ratio is defined as the ratio of minimum applied load to maximum applied load). The R-ratio was chosen slightly higher than 0 so as to have a tension-tension loading (with minimum load close to zero) on the coupons and at the same time to avoid the risk of compressive loads on the coupon.

Applied stresses were calculated dividing the applied load by the specimen net area, while the criterion to end the fatigue tests was complete specimen separation into two or more parts or no breakage until $10^{6}$ cycles. If the coupons did not break after $10^{6}$ cycles, they were considered as runouts and were not considered to derive the $\mathrm{SN}$ curve equation. All the tests were performed at a load frequency of $10 \mathrm{~Hz}$, this was chosen such that the temperature measured on the specimen surface did not exceed the test temperature by more than $5^{\circ} \mathrm{C}$ (both for high and low cycle fatigue region). Temperature monitoring was operated by a film type $\mathrm{NiCr}-\mathrm{Ni}$ thermocouple, clamped on the central part of the specimen surface. It was noticed that the rise of temperature in the surface of the specimen increases as the frequency of the applied load was increases and was about $3.3{ }^{\circ} \mathrm{C}$ at $10 \mathrm{~Hz}$, it was decided not to go for higher frequencies to avoid resonance frequency problems with the machine. All the tests shown in this paper have been performed at the same frequency of $10 \mathrm{~Hz}$.

\subsection{Numerical implementation of the MSNC}

A virtual realization of the RVE was created for each of the 0,45 and 90 degree specimens. The input is the $2^{\text {nd }}$ order orientation tensor, and the ODF is reconstructed using the method proposed by Onat and Leckie [29]. The minimum number of fibers 
needed for reliable simulation of effective properties is ascertained on the basis of a sensitivity analysis which will be described in section 3.3.

Fiber breakage during injection molding of SFRC is usually not serious due to the relatively smaller initial fiber lengths. Fiber breakage and FLD is often neglected during simulation of SFRC and a uniform length is assumed [30]. For the analysis in this paper the FLD is neglected and a constant fiber length is assumed.

The first cycle of loading is modelled within the framework of the MT formulation. Non-linear stress strain behavior of the SFRCs is simulated by modelling fiber matrix debonding as described in [22].

For the validation of the scheme, individually one of the three SN curves (corresponding to 0,45 and 90 degree coupon) is taken as the reference $\mathrm{SN}$ curve and the other two SN curves are predicted and compared with the experimental results. The validation is repeated for the three $\mathrm{SN}$ curves.

Apart from the MSNC approach, two other prevalent approaches are implemented and compared with the proposed MSNC approach. First is the UTS based scaling and the other is test based interpolation.

UTS based scaling is based on the assumption of constant proportionality between the static and fatigue strengths for different SFRCs RVE. Such an approach requires one SN curve as input and the UTS for every RVE, for this paper the UTS was experimentally derived. The ratio of the UTS and the stress to failure for a certain number of cycles to failure is calculated from the reference SN curve and its UTS. The corresponding point in the SN curve for the target RVE is then calculated by multiplying the UTS for that RVE with the above calculated ratio. This process can be repeated for as many points as is deemed necessary to generate the SN curve. 
A test based interpolation is tried by using the available $\mathrm{SN}$ curve for the 0 and 90 degree coupons. The 45 degree $\mathrm{SN}$ curve is to be estimated by linear interpolation based on the orientation angle.

The proposed MSNC approach and the other two prevalent approaches (UTS based scaling and test based interpolation) are tried at three different numbers of cycles to failures: $10^{4}, 10^{5}$ and $10^{6}$ cycles.

\subsection{Sensitivity analysis}

A SFRC with uniform random 3D fiber orientation is expected to show quasi-isotropic behavior. A certain number of inclusions (or fibers) are deemed to be high enough if both the stiffness and the fatigue strength predictions in all the directions of the isotropic SFRC are within a certain range of each other. Mathematically the size of the RVE is said to be sufficient if

$$
\frac{\text { maximum_predicted_value }}{\text { minimum_predicted_value }} \leq 1.05
$$

This approach for estimating the minimum number of inclusions for an RVE is quite common; similar approaches have been used previously [24,31] for finite element calculations and mean field homogenization schemes.

The MSNC approach is implemented using different RVEs with varying number of inclusions. The input for the calculations is taken to be the SN curve of the 0-degree coupon. SN curves are calculated for 81 different directions SFRC with uniform random 3D fiber orientation and the minimum and the maximum predicted values of the endurance strength are compared and the minimum size of the RVE is ascertained. 


\section{Results}

\subsection{Experimental results}

The UTS for the 0, 45 and 90 degree coupons was found to be 125,59 and $47 \mathrm{MPa}$ respectively. The standard deviation of the UTS for the five tests for the three sets of samples was found to be 19.0, 11.1 and 8.0 MPa respectively, which is about 15,18 and $17 \%$ of the corresponding average UTS. The average Young's modulus of the three samples was found to be 17.0, 9.4 and 8.2 GPa respectively, the standard deviation for the three sets of samples was $0.66,0.92$ and $0.60 \mathrm{GPa}$ respectively. The MT formulation predictions for the three coupons are 16.4, 9.5 and 7.8 GPa respectively.

The results of the fatigue tests are presented in Figure 5. It is seen that the strength of SFRC decreases as the orientation of the coupon increases. However this increase is not linear; the observed strength of the 45 degree coupon is only slightly higher than the strength of the 90 degree coupon and not equal to the mean of the strengths of the 0 and 90 degree coupons. The stiffness of the composite also follows a similar non-linear dependence on the orientation and was reported (for the same materials) in a recent paper [22].

The k-ratio, defined as -1/slope of the SN curve in logarithmic coordinates for the three coupons was found to be $14.4,14.6$ and 12.0 for the three cases. $T_{\sigma}$ which is defined as the ratio of the stress values at the lower and upper limit of the $90 \%$ confidence interval was also found to be similar for the three variants of coupons. The value of $T_{\sigma}$ is often seen as an indicator of the scatter observed during the fatigue tests. The observed values of $T_{\sigma}$ are equal to $1.24,1.27$ and 1.28 respectively for the 0,45 and 90 degree coupons. These values are similar to those observed by different researchers; De Monte et al. [2] reported range of $T_{\sigma}$ as 1.26-1.15, while Klimkeit et al. [32] had 1.35-1.21. 


\subsection{Sensitivity analysis}

The variation of the value of $\frac{\text { maximum_predicted_value }}{\text { minimum_predicted_value }}$ as a function of the chosen RVE size is shown below in Figure 6.

It is seen that there is a strong dependency of the stiffness and the MSNC results on the size of the RVE considered. The maximum endurance strength was seen to be 2-3 times higher than the lowest predicted endurance strength if only an RVE of about 20 fibers (inclusions) was considered. When 2265 fibers are used, the scatter in the maximum and minimum values dropped to 1.03 and 1.04 for the stiffness and the endurance strength respectively. It is confirmed from the figure that at least 1000 fibers are needed to reliably use the MSNC approach.

\subsection{MSNC approach}

The simulated results of the MSNC approach are shown in Figure 7. The experimental SN curve is denoted by solid lines and the 90 percent confidence intervals are denoted by dotted lines. Blue, red and green colors are used to depict the 0, 45 and 90 degree coupon respectively. In the figure rectangular markers indicate the predicted data points. It was observed that the predicted data points in the $\mathrm{SN}$ curves correlate well to the experimental SN curves for the three simulations. In each of the three cases the predicted SN points in the curve are within the $90 \%$ confidence interval. There is a gradual increase in error as the number of cycles to failure is decreased. This could be due to two reasons: first it is possible that the cumulative error during the micromechanics modelling increases when the applied loads are higher and second it is possible that the assumption of similar loss of stiffness is less correct at high loads. 


\subsection{MSNC approach vs. UTS based scaling}

The results of the UTS based scaling are presented in Figure 8. The results of the MSNC approach are superimposed in the same figure for comparison. It is seen that the assumption of same proportionality between the static and fatigue strength is not reasonable. The predictions of the $\mathrm{SN}$ curve by this approach are seen to be sometimes even out of the $90 \%$ confidence intervals. A closer look at the results reveals that the proportionality between the fatigue strengths and the static strengths is similar for the 0 and 90 degree coupons. This is confirmed by observing better predictions of the 90 degree $\mathrm{SN}$ curve when the 0-degree $\mathrm{SN}$ curve is taken as reference (and vice-versa). However, the predicted SN curve for 45 degree coupon (and also when 45-degree SN curve is the reference SN curve) is outside of the $90 \%$ confidence interval limit. In contrast, the predictions of the MSNC approach are significantly better and consistent for the three simulations considered. Therefore it can be concluded that the MSNC approach is more accurate as compared to the UTS based scaling.

\subsection{MSNC approach vs. test based interpolation}

Figure 9 compares the results of the test based interpolation and the MSNC approach proposed in this paper.

It is seen that a test based interpolation over-predicts the fatigue strength of the 45degree coupon. The three predicted points lie outside of the $90 \%$ confidence interval limits.

Apart from higher accuracy, the MSNC approach also provides significant benefits due to the easier testing required. Traditional interpolation based approaches require several SN curves as input. 
For interpolation, fatigue tests need to be performed on coupons with extreme FOD. Preparation of the coupons with extreme FOD is difficult and expensive. In contrast the MSNC approach needs only one SN curve as input with no requirement on the FOD.

\section{Conclusions}

A novel hybrid multi-scale method to predict the local SN curves was developed and implemented. The predictions of the MSNC approach are validated with experimental data. It was seen that the predictions of the MSNC approach are in good agreement with the experimental values. It was observed that the error in the predictions of the MSNC increases when the input number of cycles was taken to be $10^{4}$. The MSNC approach was also seen to be more accurate than the prevalent UTS based scaling and test based linear interpolation methods.

The fact that limited test data is required could be a breakthrough in view of further industrial deployment of composite solutions in industry; since collection of fatigue data is often seen as a major bottleneck for industrial deployment. The proposed method has been implemented in industrial software LMS Virtual.Lab Durability [33] part of Siemens PLM software [34].

The MSNC approach can also be used to predict the SN curve of a RVE when subjected to uni-axial tension-tension loads in different directions. This opens interesting possibilities for extensions to multi-axial loads, this aspect of the MSNC approach is within the scope of ongoing work.

An important limitation of the proposed method is that it can predict SN curves with applied load ratio close to 0 and same temperature, future work will be devoted to extending the methods to include different R-ratio as well as temperature variation. 


\section{Acknowledgement}

The authors wish to thank the IWT Vlaanderen for funding this research as a part of the project "Fatigue life prediction of random fiber composites using hybrid multi-scale modelling methods" - COMPFAT Baekeland mandate number 100689. We also want to thank PART Engineering $\mathrm{GmbH}$ for opening suitable interfaces in their software Converse to facilitate the analysis presented in this paper. Thanks also to Christophe Liefooghe and Dr. Michael Hack from Siemens Industry Software NV for useful discussions and constructive feedback. I. Verpoest and S.V. Lomov are holders of the Toray Chair in Composites at KULeuven.

\section{References}

[1] Bernasconi A, Davoli P, Basile A, Filippi A. Effect of fibre orientation on the fatigue behaviour of a short glass fibre reinforced polyamide-6. Int J Fatigue 2007;29:199-208. doi:http://dx.doi.org/10.1016/j.ijfatigue.2006.04.001.

[2] De Monte M, Moosbrugger E, Quaresimin M. Influence of temperature and thickness on the off-axis behaviour of short glass fibre reinforced polyamide 6.6 cyclic loading. Compos Part A Appl Sci Manuf 2012;41:1368-79. doi:http://dx.doi.org/10.1016/j.compositesa.2010.02.004.

[3] Klimkeit B, Nadot Y, Castagnet S, Nadot-Martin C, Dumas C, Bergamo S, et al. Multiaxial fatigue life assessment for reinforced polymers. Int J Fatigue 2011;33:766-80. doi:10.1016/j.ijfatigue.2010.12.004.

[4] Mortazavian S, Fatemi A. Fatigue behavior and modeling of short fiber reinforced polymer composites: A literature review. Int J Fatigue 2015;70:297321. doi:10.1016/j.ijfatigue.2014.10.005.

[5] Jain A, Verpoest I, Hack M, Lomov S, Adam L, Van Paepegem W. Fatigue Life Simulation on Fiber Reinforced Composites - Overview and Methods of Analysis for the Automotive Industry. SAE Int J Mater Manuf 2012;5:205-14. doi:10.4271/2012-01-0730.

[6] Mori T, Tanaka K. Average Stress in Matrix and Average Elastic Energy of Materials with Misfitting Inclusions. Acta Metall 1973;21:571-4.

[7] Vervoort S. Fatigue Analysis of polymer components with short fiber reinforcements. NAFEMS World Congr 2013. 
[8] Erhard G. Designing with polymers. Carl Hanser Publishing, Munich/Vienna; 1999.

[9] Guster C, Pinter G, Mosenbacher A, Eichlseder W. Evaluation of a Simulation Process for Fatigue Life Calculation of Short Fibre Reinforced Plastic Components. 11th Int Conf Mech Behav Mater 2011;10:6. doi:10.1016/j.proeng.2011.04.348.

[10] Mosenbacher A, Brunbauer J, Pichler PF, Guster C, Pinter G. Modelling and validation of fatigue life calculation method for short fiber reinforced injection molded parts. 16th Eur Conf Compos Mater 2014.

[11] Zago A, Springer GS. Fatigue lives of short fiber reinforced thermoplastics parts. J Reinf Plast Compos 2001;20:606-20. doi:10.1106/8u2g-bc07-3jtj-02mp.

[12] Mortazavian S, Fatemi A. Fatigue behavior and modeling of short fiber reinforced polymer composites including anisotropy and temperature effects. Int J Fatigue 2015;77:12-27. doi:10.1016/j.ijfatigue.2015.02.020.

[13] Arif MF, Saintier N, Meraghni F, Fitoussi J, Chemisky Y, Robert G. Multiscale fatigue damage characterization in short glass fiber reinforced polyamide- 66 . Compos Part B-Engineering 2014;61:55-65. doi:10.1016/j.compositesb.2014.01.019.

[14] Horst JJ, Spoormaker JL. Mechanisms of fatigue in short glass fiber reinforced polyamide 6. Polym Eng Sci 1996;36:2718-26. doi:10.1002/pen.10671.

[15] Arif MF, Meraghni F, Chemisky Y, Despringre N, Robert G. In situ damage mechanisms investigation of PA66/GF30 composite: Effect of relative humidity. Compos Part B-Engineering 2014;58:487-95. doi:10.1016/j.compositesb.2013.11.001.

[16] Fitoussi J, Bocquet M, Meraghni F. Effect of the matrix behavior on the damage of ethylene-propylene glass fiber reinforced composite subjected to high strain rate tension. Compos Part B Eng 2013;45:1181-91. doi:10.1016/j.compositesb.2012.06.011.

[17] Wyzgoski MG, Novak GE. Predicting fatigue S-N (stress-number of cycles to fail) behavior of reinforced plastics using fracture mechanics theory. J Mater Sci 2005;40:295-308. doi:10.1007/s10853-005-6082-6.

[18] Meneghetti G, Quaresimin M. Fatigue strength assessment of a short fiber composite based on the specific heat dissipation. Compos Part B Eng 2010;42:217-25. doi:http://dx.doi.org/10.1016/j.compositesb.2010.12.002.

[19] Jegou L, Marco Y, Le Saux V, Calloch S. Fast prediction of the Wohler curve from heat build-up measurements on Short Fiber Reinforced Plastic. Int J Fatigue 2013;47:259-67. doi:10.1016/j.ijfatigue.2012.09.007. 
[20] Quaresimin M, Susmel L, Talreja R. Fatigue behaviour and life assessment of composite laminates under multiaxial loadings. Int J Fatigue 2010;32:2-16.

[21] Lomov S V, Carvelli V, Verpoest I. Correlations between damage initiation thresholds in textile composites and fatigue life limits. Proc. 10th Int. Conf. Text. Compos. Recent Adv. Text. Compos., 2010, p. 475-81.

[22] Jain A, Abdin Y, Van Paepegem W, Verpoest I, Lomov S V. Effective anisotropic stiffness of inclusions with debonded interface for Eshelby-based models. Compos Struct 2015;131:692-706. doi:10.1016/j.compstruct.2015.06.007.

[23] Jain A, Van Paepegem W, Verpoest I, Lomov S V. On the loss of stiffness during cyclic loading for short fiber reinforced injection molded composites. To Be Submitt to Compos Part B n.d.

[24] Jain A, Lomov S V., Abdin Y, Verpoest I, Van Paepegem W. Pseudo-grain discretization and full Mori Tanaka formulation for random heterogeneous media: Predictive abilities for stresses in individual inclusions and the matrix. Compos Sci Technol 2013;87:86-93. doi:10.1016/j.compscitech.2013.08.009.

[25] Jain A, Abdin Y, Van Paepegem W, Verpoest I, Lomov S V. Non-symmetric stiffness tensor prediction by the Mori-Tanaka scheme - Comments on the article "Effective anisotropic stiffness of inclusions with debonded interface for Eshelby-based models, Comp. Structures 131 (2015) 692-706." Compos Struct 2015. doi:10.1016/j.compstruct.2015.08.140.

[26] Vincent M, Giroud T, Clarke A, Eberhardt C. Description and modeling of fiber orientation in injection molding of fiber reinforced thermoplastics. Polymer (Guildf) 2005;46:6719-25. doi:http://dx.doi.org/10.1016/j.polymer.2005.05.026.

[27] PLASTICS C. CAMPUS® - a material information system for the plastics industry 2014. doi:http://www.campusplastics.com/.

[28] Sigmasoft. SIGMA Engineering GmbH, Aachen. Http://www.sigmasoft.de/ 2014. doi:http://www.sigmasoft.de/.

[29] Onat ET, Leckie FA. Representation of mechanical behavior in the presence of change internal structure. Trans ASME J Appl Mech 1988;55:1-10.

[30] Kaiser JM, Stommel M. Micromechanical modeling and strength prediction of short fiber reinforced polymers. J Polym Eng 2012;32:57-66. doi:10.1515/polyeng.2011.0605.

[31] Segurado J, Llorca J, GonzÃ $j$ lez C. On the accuracy of mean-field approaches to simulate the plastic deformation of composites. Scr Mater 2002;46:525-9. doi:http://dx.doi.org/10.1016/S1359-6462(02)00027-1. 
[32] Launay A, Maitournam MH, Marco Y, Raoult I. Multiaxial fatigue models for short glass fibre reinforced polyamide. Part II: Fatigue life estimation. Int J Fatigue 2013;47:390-406. doi:http://dx.doi.org/10.1016/j.ijfatigue.2012.09.015.

[33] http://www.plm.automation.siemens.com/en_us/products/lms/virtuallab/durability/, editor. LMS Virtual.Lab Durability-Durability analysis for optimal product performance 2014.

doi:http://www.plm.automation.siemens.com/en_us/products/lms/virtuallab/durability/.

[34] www.siemens.com/plm, editor. Siemens PLM software. Www.siemens.com/plm 2014. doi:www.siemens.com/plm.

\section{List of figures}

Figure 1 A simplistic schematic representation of fatigue simulation process of SFRC

Figure 2 Typical loss of stiffness curves for SFRCs with different FOD, material is 35\% Glass Fiber reinforced PBT, coupons are machined from injection molded plates at an angle of 0, 45 and 90 degree with respect to the flow direction of the matrix.

Figure 3 A step by step algorithm of the MSNC approach to derive one data point in the SN curve for the second RVE.

Figure 4 Specimen preparation (a) plates used for injection molding. (b) The geometry of the dog bone specimen with dimensions in $\mathrm{mm}$. (c) Orientation designation of coupon.

Figure 5 SN curve of 35\% volume fraction Glass Fiber reinforced PBT, coupons are machined from injection molded plates at an angle of 0, 45 and 90 degree with respect to the flow direction of the matrix. Dotted lines indicate $90 \%$ confidence interval limits. Hollow markers indicate run outs.

Figure 6 Ratio of predicted maximum endurance strength and minimum endurance strength (black line) and stiffness $C 11$ (red line) in different directions for a quasiisotropic SFRC material made of 35\% volume fraction GF reinforced with Polybutylene terephthalate (PBT). The strength is calculated by the MSNC approach while the stiffness is calculated by the MT formulation.

Figure 7 Predictions of the MSNC approach with one of the curves (0, 45 or 90) as the reference $S N$ curve and the other two are simulated. Rectangular markers indicate the predicted data points (a) SN curve of 0-degree coupon is taken as the reference $S N$ curve (b) SN curve of 45-degree coupon is taken as the reference $S N$ curve (c) SN curve of 90-degree coupon is taken as the reference SN curve

Figure 8 Predictions of the UTS based scaling and the MSNC approach with one of the curves (0, 45 and 90 degree) as the reference $S N$ curve and the other two are simulated. Rectangular markers indicate the predicted data points by the MSNC approach, while 
circular markers indicate the predictions by the UTS based scaling. (a) SN curve of 0degree coupon is taken as the reference $S N$ curve (b) SN curve of 45-degree coupon is taken as the reference $S N$ curve (c) SN curve of 90-degree coupon is taken as the reference $S N$ curve

Figure 9 Predictions of the test based interpolation and the MSNC approach. For the test based interpolation, the 0 and 90 degree $S N$ curves are the input, while for the MSNC approach the O-degree SN curve is the reference SN curve. Rectangular markers indicate the predicted data points by the MSNC approach, while circular markers indicate the predictions by the test based interpolation. 